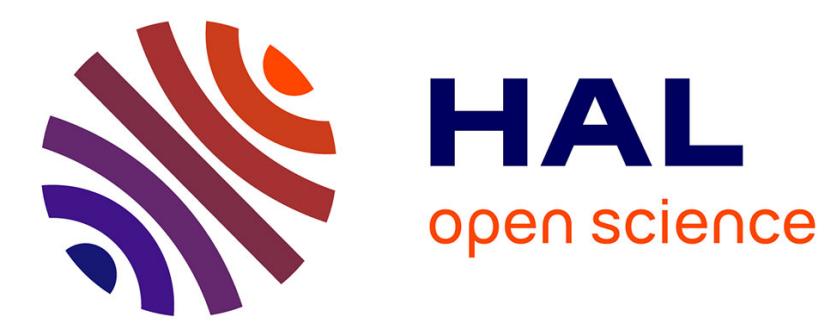

\title{
Recharging vs. Replacing Sensor Nodes Using Mobile Robots for Network Maintenance
}

Leonardo Militano, Milan Erdelj, Antonella Molinaro, Nathalie Mitton, Antonio Iera

\section{- To cite this version:}

Leonardo Militano, Milan Erdelj, Antonella Molinaro, Nathalie Mitton, Antonio Iera. Recharging vs. Replacing Sensor Nodes Using Mobile Robots for Network Maintenance. Telecommunication Systems, 2016, 10.1007/s11235-016-0145-7 . hal-01242236

\section{HAL Id: hal-01242236 \\ https://hal.inria.fr/hal-01242236}

Submitted on 7 Mar 2016

HAL is a multi-disciplinary open access archive for the deposit and dissemination of scientific research documents, whether they are published or not. The documents may come from teaching and research institutions in France or abroad, or from public or private research centers.
L'archive ouverte pluridisciplinaire HAL, est destinée au dépôt et à la diffusion de documents scientifiques de niveau recherche, publiés ou non, émanant des établissements d'enseignement et de recherche français ou étrangers, des laboratoires publics ou privés. 


\section{Telecommunication Systems \\ Recharging vs. Replacing Sensor Nodes Using Mobile Robots for Network Maintenance \\ --Manuscript Draft--}

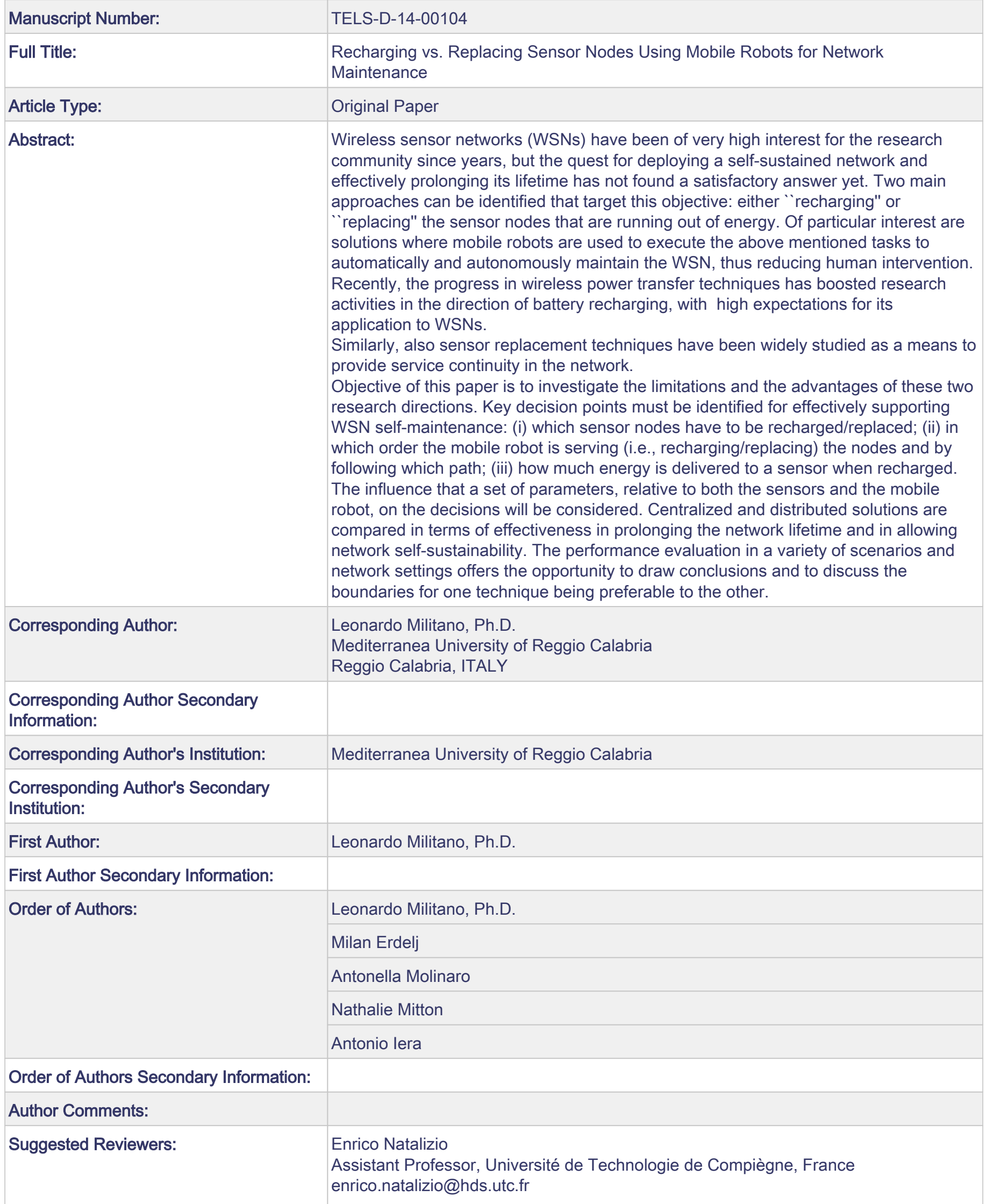


Professor Natalizio is an expert in the field of robots and sensor networks. In particular, he published several papers on the design of protocols and motion control for mobile and static devices like robots.

MJ Thinus Booysen

Senior Lecturer, Stellenbosch University, South Africa

mjbooysen@sun.ac.za

Professor Booysen is an expert, in the field of wireless sensor networks and their applications.

Valeria Loscrì

Researcher - FUN team, Inria Lille-Nord Europe, France

valeria.loscri@inria.fr

Dr. Valeria Loscrì is an expert in Wireless Sensor Networks, Robots Swarm Deployment, Robot Cooperation and Swarm Intelligence, with several papers published in this field. 


\title{
Recharging vs. Replacing Sensor Nodes Using Mobile Robots for Network Maintenance
}

\author{
Leonardo Militano • Milan Erdelj • Antonella Molinaro • Nathalie Mitton • Antonio \\ Iera
}

the date of receipt and acceptance should be inserted later

\begin{abstract}
Wireless sensor networks (WSNs) have been of very high interest for the research community since years, but the quest for deploying a self-sustained network and effectively prolonging its lifetime has not found a satisfactory answer yet. Two main approaches can be identified that target this objective: either "recharging" or "replacing" the sensor nodes that are running out of energy. Of particular interest are solutions where mobile robots are used to execute the above mentioned tasks to automatically and autonomously maintain the WSN, thus reducing human intervention. Recently, the progress in wireless power transfer techniques has boosted research activities in the direction of battery recharging, with high expectations for its application to WSNs. Similarly, also sensor replacement techniques have been widely studied as a means to provide service continuity in the network. Objective of this paper is to investigate the limitations and the advantages of these two research directions. Key decision points must be identified for effectively supporting WSN self-maintenance: (i) which sensor nodes have to be recharged/replaced; (ii) in which order the mobile robot is serving (i.e., recharging/replacing) the nodes and by following which path; (iii) how much energy is delivered to a sensor when recharged. The influence that a set of parameters, relative to both the sensors and the mobile robot, on the decisions will be considered. Centralized and distributed solutions are compared in terms of effectiveness in prolonging the network lifetime and in allowing network self-sustainability. The performance evaluation in a variety
\end{abstract}

L. Militano $\bowtie$, A. Molinaro and A. Iera

DIIES Dept., Mediterranea University of Reggio Calabria, Italy

Tel.: +390965875276

Fax: +390965875247

E-mail: name.surname@unirc.it

M. Erdelj and N. Mitton

Inria Lille-Nord Europe, France

E-mail: name.surname@inria.fr of scenarios and network settings offers the opportunity to draw conclusions and to discuss the boundaries for one technique being preferable to the other.

Keywords Wireless Sensor Networks, Mobile robots, Network maintenance, Energy recharging

\section{Introduction}

Wireless sensor networks (WSNs) are nowadays deployed for a large variety of applications and are still gaining interest in several fields [2]. The network usually consists of battery-powered wireless devices, with sensing and communication capabilities, that collect data about the environment or an event of interest (e.g., temperature, light, movement) and relay data toward a "sink" in a multi-hop manner [20]. Research in this field has been active for years, and still several open issues are waiting for valid solutions [25]. Besides the constraints in terms of processing power and storage capacity, the most important issue in WSNs is the limited battery duration of sensors and the consequent limited network lifetime.

As a consequence, WSN deployments should cope with the problem of sensor isolation and multiple deaths of nodes over the time. To manage such issues, solutions should either prevent or resolve these anomalous situations. How crucial the death of a single sensor node can be for the network, is related to the definition of network lifetime for a specific application. In this paper, we focus on the restrictive cases when the network lifetime is determined by the first dying node; in this case the objective of a network selfmaintenance solution is to prevent any single node from running out of energy. In order to succeed in this task, sensor nodes should be "refilled" with energy or "replaced" with a new node prior to their battery exhaustion. In particular, sensor recharging is a technique for sensor lifetime pro- 
longation that implies the process of directly or wirelessly recharging sensors' battery, whereas sensor replacement is the process of physical replacement of the entire sensor device or its battery unit. Noteworthy, when WSNs are being deployed in hardly accessible areas, or where human intervention is costly to carry out recharging/replacement tasks, the use of mobile robots for automatic and autonomous WSN maintenance becomes of high interest; this is the reference scenario for this paper.

The research in the direction of sensor recharging has been fueled by the recent evolution in wireless power transfer techniques [5]-[6]. The recharging solutions still have some issues to solve, mainly related to the best route the mobile robot has to follow to refill the battery of exhausting sensors. On the other hand, the alternative technique of sensor replacement has additional practical limitations related to the number of sensors a mobile robot can carry around simultaneously. This aspect can be very limiting in applications where the sensing hardware is not dimensionally small.

Solutions will be proposed for answering key questions such as: (i) which sensor nodes are to be recharged/replaced; (ii) which order the robot is going to follow to serve (i.e., recharge or replace) the nodes and which delivery route is chosen; (iii) how much energy is delivered to a sensor when recharged. Several parameters (such as battery capacity of the mobile robot, time needed to recharge the nodes, number of nodes in the network, battery power consumption, and network topology) influence the effective system design. The most important among them will be duly taken into account in our research.

The remainder of the paper is organized as follows. The next section gives an overview of the related work relevant to node recharging and replacement techniques to prolong the network lifetime. In Section 4 a detailed overview and definition of system settings, parameters of interest, and objectives for the present work is given. Sections 5 and 6 describe the proposed solutions to the raised problem, when either recharging or replacement techniques are adopted. In Section 7 a performance evaluation for the proposed solutions is presented, and final remarks in Section 8.

\section{Related Work}

Energy conservation solutions in WSN is a widely investigated topic in the literature [7], [3], [1], [11], [24] as the limited energy storage capacity of sensors requires solutions that either solve or alleviate the impact on the service continuity in a WSN. Besides the two approaches discussed in this paper, also energy harvesting techniques that convert ambient energy (for instance, solar, wind, thermal or vibration energy [15]) into electrical energy could be applied. However, even if the results seem to be promising, in many cases their success is limited due to the environment dependent technology and the strong requirements for practical deployment.

\subsection{Node Recharging Solutions}

The ability to transfer electric power from one storage device to another one [5] or to multiple devices [6] without any plug or wire, and in non-line-of-sight conditions, is indeed revolutionary. In fact, these novel techniques pave the way for a plethora of innovative applications and service when recharging of battery-driven devices is of vital importance for the system sustainability (e.g., border surveillance, security monitoring, agents-based patrolling, real-time fire detection).

Particular attention has been devoted to these techniques by the WSN research community, where self-sustainability and network lifetime are topics of utmost importance. This is witnessed, for instance, by recent works in [14], [22] and [23] where the authors propose very powerful solutions for network self-sustainability based on wireless power transfer between devices. In particular, in [14] and [22] the authors present a system model where a recharging vehicle visits all the nodes in the network to fully recharge them. In the proposed solution, using a vehicle instead of a mobile robot gives some degrees of human intervention in the network. An optimization problem is modeled to maximize the time the vehicle is not active over the time it is driving around the network to recharge the nodes. A key assumption for the proposed model is that the vehicle has infinite energy to recharge the nodes; this assumption guarantees that the vehicle fully recharges the whole network in every visiting cycle. In their contributions the authors show that, under certain conditions and network settings, the network is actually "immortal" in the sense that no sensor is ever running out of battery, which is a very promising result.

In [23] the problem is extended by considering the possibility to divide the network area into cells and recharge more nodes simultaneously. While this solution clearly improves the network performance, still the scalability remains an issue since for larger number of nodes always a limiting condition exists where a single vehicle is not able to find a solution that guarantees the network immortality.

The wireless power transfer technology has been studied in other contributions as well. In particular, in [12] with the objective to prolong the network lifetime, the effects of different configurations and design parameters are studied in a scenario where a robot delivers energy to single sensors. The robot has a limited battery capacity and it has to find the optimal charging sequence, that is the sequence of nodes to visit and recharge. At the same time, the amount of time to recharge sensors is not fixed and needs to be defined. Various traveling and charging algorithms are compared show- 
ing the beneficial effect of simultaneously considering the robot movement plan and the residual energy of each sensor nodes in every instant of time. Nevertheless, prolonging the network lifetime and immortality is not guaranteed; this result showing that further investigation in this field is strongly needed.

\subsection{Node Replacement Solutions}

Node replacement is a valid alternative to be considered whenever a sensor node fails due to different reasons (e.g., running out of energy, hardware crash, etc.). A viable approach to node replacement is to fill sensing holes by relocating redundant nodes in the network. To implement this solution either sensors mobility is required [18] or a mobile robot can be used to discover holes and replace out-of-work nodes in the network [8]. A challenging issue for the latter solutions is to allow mobile robots to precisely navigate toward the interested sensor nodes. This aspect is analyzed in [13], where a solution based on the received signal strength allows to determine the direction taken by the mobile robot. A further alternative to cope with sensing holes is proposed in [9], where a small number of mobile robots is used to replace failed sensors with new ones. This is particularly interesting when nodes are static and have no possibility to relocate autonomously. In particular, the authors propose both centralized and distributed algorithms for detecting and reporting sensor failures, and coordinating the movement of robots to minimize the motion energy and the messaging overhead.

A different approach is proposed in [17] and [16], where a node reclamation and replacement strategy is presented. A mobile repairman (a robot or a human labor) periodically replaces sensors with low or null energy with fully charged ones. The retrieved sensors are then recharged at a base station. In particular, in [17] the reference scenario considers multiple sensors near the same location and several sensing locations in the system. The solution is based on a two-tier scheduling; the global-tier scheduling calculates the travel schedule and the number of sensors to be reclaimed/replaced; the local-tier scheduling regards the local activity scheduling of the sensors in the group. While the two-tier solution is interesting in general, for the scenario studied in the paper, the sensor nodes are allowed to die before being replaced, as long as the sensing quality in the group they belong to meets the system requirements. In [16] the same research is extended to consider also network area coverage instead of point coverage, where single points of interest are monitored within a network, as studied in [17].

\section{Motivations and Objectives}

As described in the previous sections, both node recharging and node replacement techniques seem to be valid approaches to prolong the network lifetime in WSNs. An investigation on the key aspects, on the limitations and the advantages of these two research directions would offer the network designer useful information to deploy and manage a WSN. The focus of this paper is limited to a single mobile robot moving around the network to carry out the task of recharging/replacing energy endangered sensors. The scope of the analysis is oriented on highlighting the different solutions and approaches that can be proposed to model a WSN maintenance based on either of these techniques.

A first classification of the possible solutions discerns between centralized and distributed approaches. This classification refers to the recharge/replace decisions taken by the mobile robot. With a centralized approach decisions are taken at the base station, which then provides the mobile robot with instructions on the best way to visit the nodes that need assistance. This implies some information processing at the base station, as it is supposed to have full-knowledge of the state of the network. This is a reasonable assumption under the hypothesis of sending energy state information from the sensors to the base station through the WSN. A distributed approach, instead, leaves space to the robot to implement its own method to visit the nodes. These choices will be performed step-by-step, based on the information the mobile robot collects from sensors in its sensing range and current energy conditions of nodes and mobile robot.

For any of the approaches to implement, a WSN maintenance solution is required to answer the following questions:

1. Which nodes will be visited by the robot for maintenance purposes?

2. In which order should the nodes be visited by the robot?

3. How much energy should each visited node receive from the robot? Only in case of energy recharging solutions, the amount of energy to be transferred to the sensor nodes to prolong the network lifetime shall be defined.

In particular, the first two questions yield for any of the considered approaches, whereas the third question refers only to recharging techniques. Examples of study cases that can be identified are: (i) the mobile robot has the possibility to reach and fully serve (when recharging this means fully recharge the battery of the sensor) all nodes requiring assistance, (ii) the mobile robot has not the possibility to reach and serve all nodes requiring assistance due to its low energy for movement or long distances between the sensors, (iii) the mobile robot can reach and serve all nodes requiring assistance, but has not the possibility to replace all nodes or recharge the nodes fully due to low energy or low number of sensors for replacement available, (iv) the time available 
to the mobile robot for recharging the sensors is enough to visit all sensors requiring assistance, but they cannot be fully recharged.

Solutions will be proposed for any of the study cases, and the performance evaluation presented in this paper aims at evaluating the proposals in a variety of scenarios and network settings. In particular, the boundaries for one technique being preferable to the other will be investigated, whereas the choices relevant to robot movement and served node selection will be compared and discussed.

\section{Reference System Overview and Assumptions}

In this section, basic notions and background information about the considered WSN are introduced to support and guide the reader through our analysis. An assumption that simplifies the analysis is to consider heterogeneous sensor networks, with static sensors of different types (sensing temperature, luminosity, movement, radiation, etc.) but with equa power consumption law and recharge rate. We assume that all nodes start with the same energy level, while their energy consumption depends on the sensing and message broadcasting activities within the WSN. Furthermore, each sensor piggybacks energy status and consumption information jointly with the data in the messages transmitted to the sink (or base station).

\subsection{Sensors}

We refer to the $i^{t h}$ sensor node with notation $S_{i}$, and to its position in the network by its Euclidean coordinates $S_{i}\left(x_{i}, y_{i}\right)$. Given two sensor nodes $S_{u}$ and $S_{v}$ with positions $S_{u}\left(x_{u}, y_{u}\right)$ and $S_{v}\left(x_{v}, y_{v}\right)$, we say that sensors $S_{u}$ and $S_{v}$ can communicate if their Euclidean distance $d\left(S_{u}, S_{v}\right)$ is less than the sensor communication range $r^{c}$ that is supposed to be the same for all the sensors. Consequently, the WSN can be represented as a graph $\mathcal{G}(\mathcal{V}, \mathcal{E})$, where $\mathcal{V}$ is the set of sensor nodes (including the sink) and $\mathcal{E}$ is the set of edges, $\mathcal{E}=\left\{\left(S_{u}, S_{v}\right) \in \mathcal{V} \mid S_{u} \neq S_{v} \wedge d\left(S_{u}, S_{v}\right) \leq r^{c}\right\}$. We denote by $|\mathcal{V}|$ the number of sensors in $\mathcal{V}$.

In order to have a realistic evaluation of the energy consumption in the network, we consider a WSN where every sensor $S_{i}$ generates a message about the event it has sensed with a frequency $F_{i}$. After the message is generated, it will be transmitted toward the data sink in a multi-hop manner with a sensor transmitting at instant $t$ at a rate $T_{i}(t)$. In terms of power consumption, sensing and transmitting activities of sensor $i$ are characterized by $P_{i}^{s}$ and $P_{i}^{t}$, respectively. Any multi-hop transmission in a WSN requires message relaying policies and represents an important source of sensor energy consumption.
Table 1 Notations for sensor node $S_{i}$.

\begin{tabular}{|l|l|}
\hline Notation & Explanation \\
\hline \hline$S_{i}\left(x_{i}, y_{i}\right)$ & Position in Euclidean coordinates \\
\hline$r^{c}$ & Communication range \\
\hline$F_{i}$ & Information sampling rate \\
\hline$T_{i}(t)$ & Transmission rate in instant $t$ \\
\hline$P_{i}^{s}$ & Sensing power consumption \\
\hline$P_{i}^{t}$ & Transmission power consumption \\
\hline$L_{i}(t)$ & Expected lifetime at instant $t$ \\
\hline$E_{i}(t)$ & Residual energy at time $t$ \\
\hline$E_{\text {max }}$ & Maximum energy that can be stored in the battery \\
\hline $\operatorname{Emin}_{i}$ & Minimum energy threshold for being active \\
\hline$R^{c h}$ & Battery recharging rate (assumed equal for all sensors) \\
\hline$P_{i}(t)$ & Total power consumption equal to: $P_{i}^{s} F_{i}+P_{i}^{t} T_{i}(t)$ \\
\hline
\end{tabular}

During network operations, some of the sensors will inevitably run out of the energy and, thus, possibly jeopardize the whole network functional behavior. The expected sensor lifetime at time $t$, i.e., $L_{i}(t)$, is defined as the time between the sensor start-up, i.e., its "birth", when its current battery is fully charged, $E_{i}(t)=E_{\max }$, until its "death", when its energy level falls below the minimum energy threshold $\operatorname{Emin}_{i}$. Similarly, the network lifetime is defined as the time between the "birth" of the sensor network until the first interruption of the sensing activity of a single sensor in the network $[4,19]$.

The Recharging procedure of a generic sensor $i$ is characterized by a recharging duration $T_{i}^{c h}$ that depends on the amount of energy transferred to the sensors. In particular, to fully recharge a sensor with maximum energy capacity $\operatorname{Emax}_{i}$, current battery energy level $E_{i}(t)$ at time $t$, and a recharging rate $R^{c h}$ of the battery, we have that:

$$
T_{i}^{c h}=\frac{\operatorname{Emax}_{i}-E_{i}(t)}{R^{c h}} .
$$

Replacement is, instead, characterized by the time $T^{r}$ required by the implemented replacement technique/process of the mobile robot. Table 1 summarizes the further information of interest relevant to a sensor node $i$.

In the context of this paper, the specific technique adopted to recharge or replace is not very relevant. In fact, the parameter that actually makes the difference between the two techniques is the required time to carry out the task. For the implementation of both types of analysed techniques, we consider the use of a mobile robot (instead of a vehicle or a human) so that human intervention is minimized.

\subsection{Mobile robots}

A mobile robot is a mobile robotic platform capable of moving across the positions of target sensor nodes for maintenance purposes. In this paper a mobile robot is designated by the notation $R$ and it is characterized by its Euclidean position within the network $R(x, y)$ and a sensing range $r^{s}$. We 
assume that the base station is the energy source for the mobile robot and the sensors: the robot can be charged directly by visiting the base station, while sensor nodes are charged indirectly via the mobile robot. Moreover, we assume that the time needed for a robot to recharge/replace its own energy sources at the base station $T^{\text {replace }}$ is set to zero. This can for instance be implemented by swapping the robot at the base station where a fully charged robot is waiting to take the place of the exhausted incoming robot.

Table 2 Notations for mobile robot $R$.

\begin{tabular}{|l|l|}
\hline Notation & Explanation \\
\hline \hline$R(x, y)$ & Position in Euclidean coordinates \\
\hline$E m a x^{m}$ & Total energy available for movement \\
\hline$E^{m}(t)$ & Remaining energy for movement at time $t$ \\
\hline$E m a x^{r}$ & Total energy available for recharging \\
\hline$E^{r}(t)$ & Remaining energy for recharging at time $t$ \\
\hline$N m a x^{r}$ & Maximum number of sensor nodes for replacement \\
\hline$T^{r}$ & Time needed to replace a sensor node \\
\hline$P^{m}$ & Power consumption for movement \\
\hline$V^{m}$ & Movement speed \\
\hline$P^{r}$ & Power consumption for recharging \\
\hline$T^{\text {replace }}$ & Time for own battery recharge/replacement \\
\hline$r^{s}$ & Sensing range \\
\hline
\end{tabular}

The mobile robot is characterized by a movement speed $V^{m}$ and a power consumption for movement $P^{m}$. When recharging a sensor node, the mobile robot has a power consumption $P^{r}$. A further contribution to the power consumption of a mobile robot is the operation power consumption, but this is very small and set to zero for simplicity in the analysis. A distinction has to be made between the cases when a robot uses the same or a different energy source for movement and recharging. The choice in this paper is to keep the two energy sources separated, as the energy consumption due to the movement of a mobile robot is not negligible. In particular, a mobile robot will have a distinct amount of energy available for movement and recharging, i.e., Emax ${ }^{m}$ and $\operatorname{Emax}^{r}$ respectively. Thus, when modeling any maintenance solution, the energy constraints set by the current remaining energy at time $t$ for recharging and movement should be considered separately, i.e., $E^{r}(t)$ and $E^{m}(t)$ respectively. Instead, when a replacement technique is implemented, the energy dedicated to recharging is not of interest. In particular, this constraint is replaced by a limitation on the number of node replacements nodes max $^{r}$ the mobile robot can perform, whereas $T^{r}$ is the time needed to replace a sensor node. All assumed robot features are summarized in Table 2.

\section{Node Recharging Techniques}

In all the solutions presented next, a mobile robot reaches the exact position of a sensor in the network. Moreover, we assume that when the robot stops at a recharging position only the targeted sensor will be recharged. With this assumption we will obtain a lower bound performance for any proposed solution. In fact, in dense scenarios where multiple sensors can simultaneously be recharged by the mobile robot, the performances in terms of network lifetime prolonging potentialities are expected to be higher as discussed in [23].

\subsection{Centralized Approach for Node Recharging}

According to this approach, a mobile robot collects at the base station all the instructions it has to fulfill in the next maintenance cycle, including the exact path to follow to recharge the nodes in need of assistance. The input information taken into account is: the set of energy- endangered nodes, i.e., the nodes that will die if not recharged in the next maintenance cycle; the amount of energy spent on recharging a sensor; and the energy spent by the robot to move toward the sensor.

Noteworthy, finding an optimal tour where each energy endangered node is visited once per tour and provided with the required energy is an NP-Hard problem, as it can be modeled as a well-known Traveling Salesman Problem (TSP) [10]. Therefore, we propose heuristic algorithms to solve the problem. In view of this, the energy constraints set by the mobile robot for movement and the energy to be transferred to the sensors are mapped onto the corresponding required time. In particular, we will consider the total time available to the robot to move as the fraction of the maximum energy available for movement and the power consumption for movement: $\frac{\operatorname{Emax}^{m}}{P^{m}}$. Similarly, the total time available to recharge the sensor nodes is evaluated as the fraction of the maximum energy available to recharge and the power consumption in recharging: $\frac{\operatorname{Emax}^{r}}{P^{r}}$.

Considering the problem in terms of time, we introduce a key parameter for the centralized approaches. In particular, we define a maintenance cycle duration $T$ as the time between two consecutive visits of the robot at the base station. This time is available to the robot to perform the maintenance tasks and it will be split into the time needed for moving operations to reach the sensor nodes, and the time needed fr recharging operations. The proposed solutions are also requested to define how this time $T$ is used by the mobile robot.

To the purpose, any recharging centralized technique operates by answering the three key questions listed in Section 3 as detailed in the following and summarized in Algorithm (1). 
Table 3 Sensor node list provided to the robot by the data sink at time $t$

\begin{tabular}{|c|c|c|c|c|c|c|c|}
\hline Sensor & $\begin{array}{c}\text { Euclidean } \\
\text { coordinates }\end{array}$ & $\begin{array}{c}\text { Minimum } \\
\text { energy }\end{array}$ & $\begin{array}{c}\text { Maximum } \\
\text { energy }\end{array}$ & $\begin{array}{c}\text { Residual } \\
\text { energy }\end{array}$ & $\begin{array}{c}\text { Mean power } \\
\text { consumption }\end{array}$ & $\begin{array}{c}\text { Expected } \\
\text { lifetime }\end{array}$ & $\begin{array}{c}\text { Battery } \\
\text { recharge rate }\end{array}$ \\
\hline \hline 1 & $S_{1}\left(x_{1}, y_{1}\right)$ & $\operatorname{Emin}_{1}$ & $\operatorname{Emax}_{1}$ & $E_{1}(t)$ & $P_{1}=\left(E_{1}(t-T)-E_{1}(t)\right) / T$ & $L_{1}(t)$ & $R^{c h}$ \\
\hline 2 & $S_{2}\left(x_{2}, y_{2}\right)$ & $\operatorname{Emin}_{2}$ & $\operatorname{Emax}_{2}$ & $E_{2}(t)$ & $P_{2}=\left(E_{2}(t-T)-E_{2}(t)\right) / T$ & $L_{2}(t)$ & $R^{c h}$ \\
\hline$\ldots$ & $\ldots$ & $\ldots$ & $\ldots$ & $\ldots$ & $\ldots$ & $\ldots$ & $\ldots$ \\
\hline $\mathrm{n}$ & $S_{n}\left(x_{n}, y_{n}\right)$ & $\operatorname{Emin}_{n}$ & $\operatorname{Emax}_{n}$ & $E_{n}(t)$ & $P_{n}=\left(E_{n}(t-T)-E_{n}(t)\right) / T$ & $L_{n}(t)$ & $R^{c h}$ \\
\hline
\end{tabular}

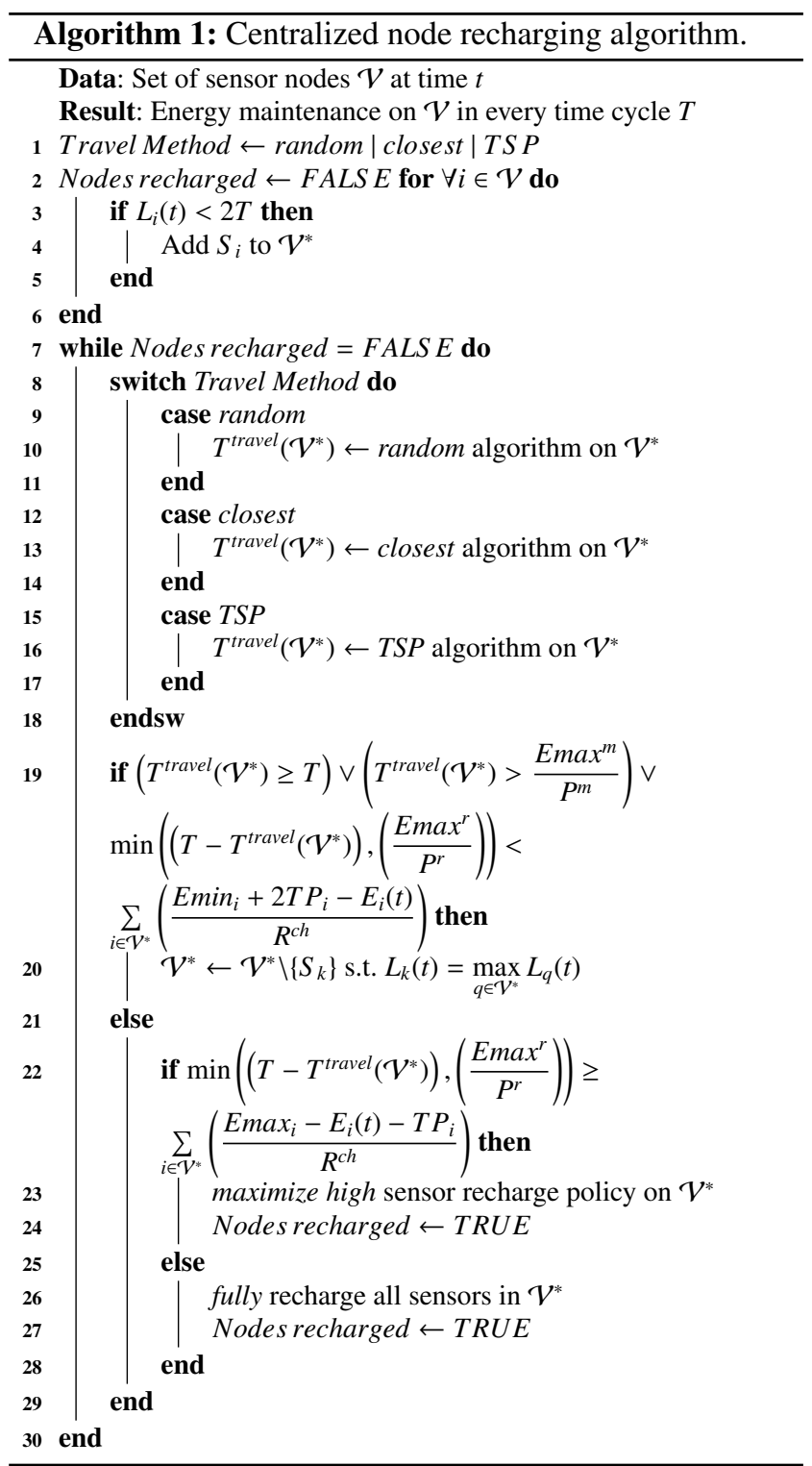

\subsubsection{Nodes visited for maintenance}

Based on the collected information, the base station, identifies the set of sensors that need to be recharged in the next time window of duration $T$. This set of nodes is denoted by $\mathcal{V}^{*}$. In particular, a sensor is considered as endangered, and denoted by $S_{i}^{*}$, if its energy level in the next two time windows $E_{i}(t+2 T)$ is expected to drop under the threshold
$\operatorname{Emin}_{i}$. This means that a sensor is added to $\mathcal{V}^{*}$ if its expected lifetime $L_{i}(t)$ is lower than $2 T$ (please refer to lines 1-7 in Algorithm 1). The information collected by the base station about nodes includes, therefore, also the mean power consumption in the previous time window of duration $T$, see Table 3. Based on this information, the base station estimates the expected lifetime of each sensor in the network.

\subsubsection{Node visiting order}

Once the set of endangered nodes is found, the path to visit all the selected sensors within the time frame $T$ must be defined for the mobile robot. We propose three possible path selection policies (please refer to lines 9-19 in Algorithm 1):

- random: the order of the sensors to visit is randomly chosen;

- closest: the order of the sensors to visit is built by iteratively choosing the node with the lowest product of the Euclidean distance from the current position and the expected lifetime of the node;

- TSP: the path to cover is defined by an implementation of the well-known Traveling Salesman Problem (TSP) [10] solution.

As an example, in Fig. 1, an implementation of the three solutions is plotted for a sample study case scenario with eight sensors.

As a consequence of the definition of the mobile robot path, the estimation of the time $T^{\text {travel }}\left(\mathcal{V}^{*}\right)$ needed to cover the selected path is possible. Moreover, also the required energy for moving along the selected path can estimated: $E^{\text {visit }}\left(\mathcal{V}^{*}\right)=T^{\text {travel }}\left(\mathcal{V}^{*}\right) \cdot P^{m}$. These values are useful to answer the third question and identify the possible study cases, as detailed next.

\subsubsection{The amount of energy required by each visited node}

For a successful energy recharging solution at least the minimum energy should be transferred to all the endangered nodes so that they stay alive during the next time cycle of duration $T$. When considering the energy constraints of the mobile robot (both for recharging and for movement) and those of the sensor nodes, four different study cases are identified. To best identify them, all parameters are analyzed in terms of time, i.e., time to travel, time to recharge a sensor 


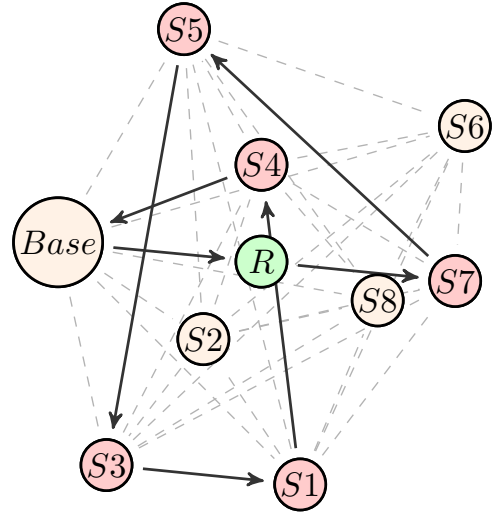

(a) Random path selection

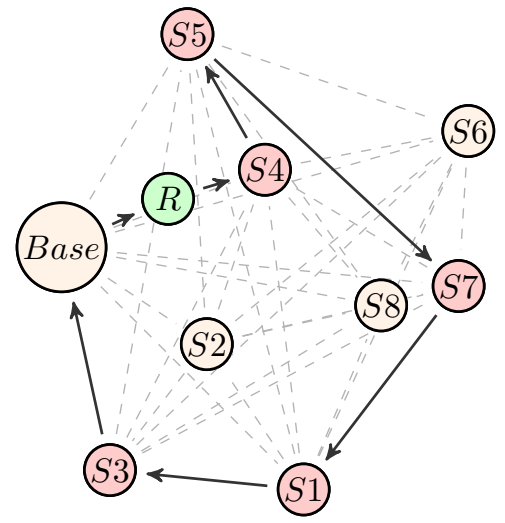

(b) Path selection based on closest node

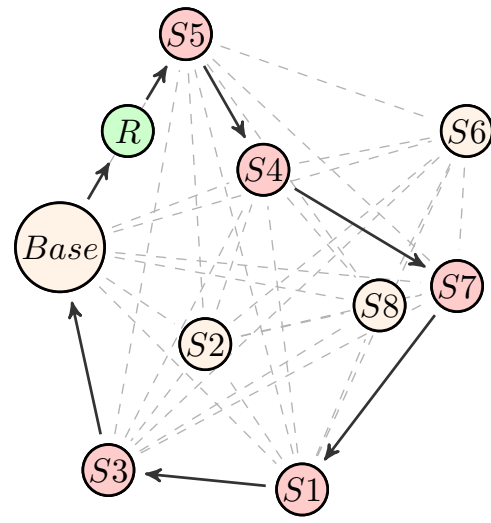

(c) Path selection based on Traveling Salesman Problem solution

Fig. 1 The robot calculates the path to follow considering only the sensors that need recharging. If sensors $S_{2}, S_{6}$ and $S_{8}$ do not require additional energy, the robot follows a selected path providing the remaining sensors with energy.

with minimum or maximum energy, time corresponding to the energy available at the robot for moving or recharging.

- Case 1: the time $T^{\text {travel }}\left(\mathcal{V}^{*}\right)$ to visit all sensors in $\mathcal{V}^{*}$ is larger then total cycle time $T$ or the required energy is larger than the energy available for movement at the robot:

$$
\left(T^{\text {travel }}\left(\mathcal{V}^{*}\right) \geq T\right) \vee\left(T^{\text {travel }}\left(\mathcal{V}^{*}\right)>\frac{\operatorname{Emax}^{m}}{P^{m}}\right) .
$$

In this case, we propose to iteratively remove one of the sensors to be recharged until the feasibility conditions are met and the reduced set of sensors can be recharged, in the hope that the removed nodes will survive (e.g., the lifetime value was a pessimistic estimation of the real value). In particular, the less critical nodes with the highest estimated lifetime $L_{i}(t)$ are removed from the set of nodes to recharge $\mathcal{V}^{*}$ (please refer to lines 20-21 in Algorithm 1).

- Case 2: the available time to recharge the sensors in $\mathcal{V}^{*}$, is not enough to recharge them all with the minimum required energy ${ }^{1}$. This condition is computed by evaluating whether the sensors will have at time $t+2 T$ at least $\operatorname{Emin}_{i}$ energy $^{2}$ :

$$
\min \left(\left(T-T^{\text {travel }}\left(\mathcal{V}^{*}\right)\right),\left(\frac{\text { maxx }^{r}}{P^{r}}\right)\right)<\sum_{i \in \mathcal{V}^{*}}\left(\frac{\operatorname{Emin}_{i}+2 T P_{i}-E_{i}(t)}{R^{c h}}\right) .
$$

Also in this case, we propose to remove iteratively the node with the highest estimated lifetime $L_{i}(t)$ from the

\footnotetext{
1 The time available to recharge a sensor is limited by the lower of the values of two parameters: the spared time in cycle $T$ during the robot travel, the energy for recharging (mapped onto a time value) available in the robots battery.

2 A $2 T$ time is considered to cover the case of a sensor recharged at the beginning of one cycle and then at the end of the next cycle.
}

set of nodes to recharge $\mathcal{V}^{*}$ until the feasibility conditions are met (lines 20-21 in Algorithm 1).

- Case 3: the time available to recharge the sensors in $\mathcal{V}^{*}$, is enough to recharge all the nodes fully ${ }^{1}$ :

$\min \left(\left(T-T^{\text {travel }}\left(\mathcal{V}^{*}\right)\right),\left(\frac{\text { Emax }^{r}}{P^{r}}\right)\right) \geq \sum_{i \in \mathcal{V}^{*}}\left(\frac{\operatorname{Emax}_{i}-E_{i}(t)-T P_{i}}{R^{c h}}\right)$.

In this case the robot will visit all sensors and recharge them completely (please refer to lines 26-28 in Algorithm 1).

- Case 4: the time available to recharge the sensors in $\mathcal{V}^{*}$, is enough to recharge them all with minimum required energy ${ }^{1}$, i.e., at time $t+2 T$ the energy in the battery will be at least $\operatorname{Emin}_{i}$, but it is not enough to recharge them all fully:

$$
\begin{gathered}
\sum_{i \in \mathcal{V}^{*}}\left(\frac{\text { Emin }_{i}+2 T P_{i}-E_{i}(t)}{R^{c h}}\right) \leq \min \left(\left(T-T^{\text {travel }}\left(\mathcal{V}^{*}\right)\right),\left(\frac{\text { Emax }^{r}}{P^{r}}\right)\right) \\
\leq \sum_{i \in \mathcal{V}^{*}}\left(\frac{\text { Emax }_{i}-E_{i}(t)-T P_{i}}{R^{c h}}\right) .
\end{gathered}
$$

In this case a policy is required to decide how to distribute the remaining energy among the sensors (see lines 23-25 in Algorithm 1). More details on the latter aspect are given in Section 5.1.3.

Energy allocation solutions for Case 4 in centralized recharging solutions: This case is particularly interesting as several possible policies can be adopted. A simple approach is to equally distribute the energy (recharge time) among the sensors. Differently, in the view of maximizing the number of time windows in which they are not endangered, an alternative approach is to recharge some nodes fully, while guaranteeing the minimum energy to the others. Parameters such as the power consumption or the expected lifetime may drive the choice of the nodes to be fully recharged. A further alternative is to adopt a weighted function that guarantees the 
minimum to all sensors, like in equation (1):

$$
\begin{array}{r}
T_{i}^{c h}=\left(\frac{\operatorname{Emin}_{i}+2 T P_{i}-E_{i}(t)}{R^{c h}}\right)+ \\
\frac{\omega_{i}}{\sum_{i \in \mathcal{V}^{*}} \omega_{i}}\left(\left(T-T^{\text {travel }}\left(\mathcal{V}^{*}\right)\right)-\sum_{i \in \mathcal{V}^{*}}\left(\frac{\operatorname{Emin}_{i}+2 T P_{i}-E_{i}(t)}{R^{c h}}\right)\right),
\end{array}
$$

under the constraint that a sensor cannot be recharged with more energy than its maximum capacity and by defining $\omega_{i}$ as the weight for a sensor node proportional, e.g., to its importance, power consumption, or lifetime.

An analysis on sample random networks has been performed to have some indications on the best policy to implement. We compared solutions where the energy is shared equally, or it is allocated proportionally to the lifetime according to weighted function (1), as well as where some nodes are fully recharged while others are not, according to their expected lifetime $L_{i}(t)$. From this analysis we observed that, especially for small number of nodes, the most performing solution is the one we hereinafter call maximize high solution. According to it, the residual energy is used to fully recharge as many sensors as possible , by following an ordering from the highest expected lifetime $L_{i}(t)$ to the lowest expected lifetime of the sensors.

\subsection{Distributed Approach for Node Recharging}

In the distributed approach, adopted solutions are not decided by the base station, and the robot will autonomously decide how to recharge the sensor nodes. This stepwise choice will be based on the information (updated over the time) it collects from the sensors in its sensing range.

\subsubsection{Nodes visited for maintenance}

A new node is visited if and only if the robot energy available for movement is enough to visit the selected node and reach the base station, if needed, to recharge itself.

\subsubsection{Node visiting order}

We propose three different algorithms for the selection of the next sensor to recharge. A graphical overview of the proposed solutions is given in Fig. 2, where in a sample network scenario with eight sensors the mobile robot at the base station makes the first node selection. The proposed solutions are listed below:

- random: the next sensor the mobile robot will visit is randomly chosen based on the known information;

- minenergy: the sensor with the current minimum energy level is selected as the next node to visit;

- minlife: the sensor with the current minimum expected lifetime is selected as the next node to visit.

\subsubsection{The amount of energy required by each visited node}

In all of the cases listed above, when a sensor is visited the robot will recharge it fully. Moreover, at each time instant it is checked if the energy needed to recharge the selected node is available and if the residual energy for moving in the robot is enough to reach the selected node and then go back to the base station. If both conditions are not met, then the mobile robot will go back to the base station to recharge/replace its own power supplies.

The distributed recharge algorithm is summarized in Algorithm (2). In particular, by starting from the base station, the next node to visit derives from the adopted method random, minenergy or minlife (see lines 1-22 in Algorithm 2). Then, the energy the robot requires to move toward the selected node and move back to the base station is computed (see line 23). Also the energy to fully recharge the targeted node is computed (see line 24). If the power available at the mobile robot is not enough to satisfy both energy requirements, then the selected node is not served and the mobile robot moves back to the base station to replace its own power supply (see lines 25-31). Otherwise, the selected node is visited and fully recharged, and the mobile robot is ready to choose the next node to visit (see lines 33-35 in Algorithm 2).

\section{Node replacement techniques}

When analyzing the node replacement techniques, two main differences must be considered with respect to the recharging based solutions. The first one is that the time needed to replace a sensor $T^{r}$ is generally much lower than the time needed to fully recharge. The second main difference is in the constraint to be set on the maximum number of sensors a mobile robot can carry $N \max ^{r}$, which replaces the maximum amount of charging energy a robot can spend $E$ max $^{r}$.

\subsection{Centralized Approach for Node Replacement}

Also for sensor nodes replacements, the mobile robot will collect all the instructions about the tasks to fulfil in the next maintenance cycle of duration $T$ at the base station. The analysis on the mobile robot energy needed for movement will, again, be performed based on the corresponding time duration. When replacing techniques are implemented, only the following two issues need to be addressed.

\subsubsection{Nodes visited for maintenance}

Similarly to the recharge based solutions, the base station collects all the information needed to find the set of sensors $V^{*}$ to substitute in the next time window of duration $T$. Also 


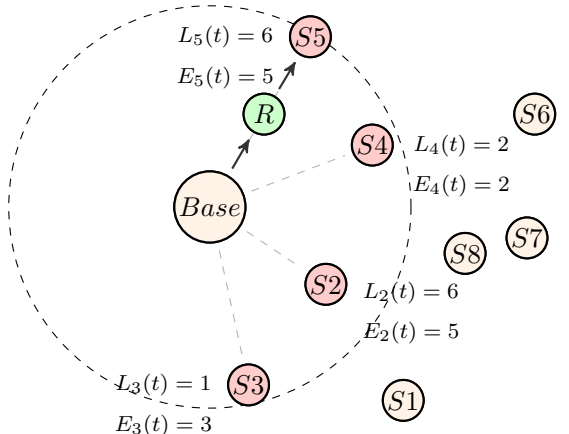

(a) Random next node selection

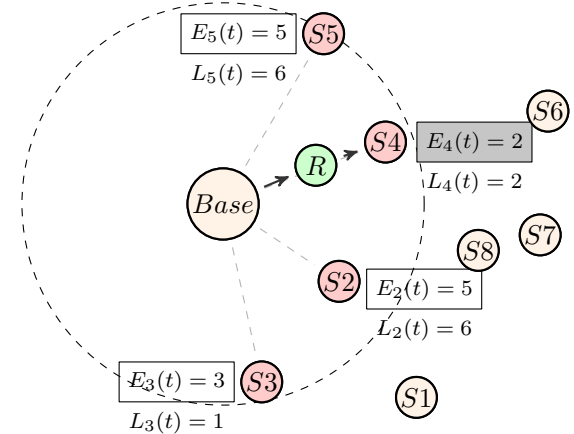

(b) Next node selection based on energy level

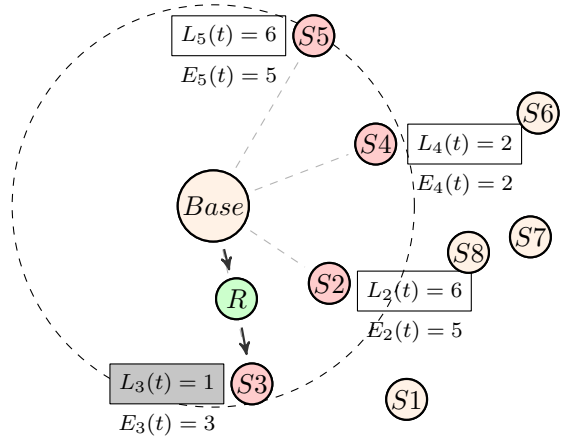

(c) Next node selection based on expected lifetime

Fig. 2 The path to follow for the robot is calculated stepwise based on the knowledge gathered from the coverage range of the robot.

in this case, a sensor $S_{i}$ is considered as endangered, denoted by $S_{i}^{*}$, if its energy level in the next two time windows $E_{i}(t+$ $2 T$ ) is expected to drop under the threshold $\operatorname{Emin}_{i}$.

\subsubsection{Node visiting order}

Also for the replacement case, three different solutions discussed in section 5.1 are considered for the traveling path of the mobile robot, namely random, closest and TSP.

In Algorithm (3) all the steps of the replacement algorithm are illustrated. Note that the occurring cases for replacement solutions differ from those of the recharging solution and are listed below:

- Case 1: either the time to visit all nodes in $\mathcal{V}^{*}$ is larger than total cycle time $T$ or the energy required to cover the complete distance is more than that available in the robot (the condition is also here expressed in terms of time needed):

$$
\left(T^{\text {travel }}\left(\mathcal{V}^{*}\right) \geq T\right) \vee\left(T^{\text {travel }}\left(\mathcal{V}^{*}\right)>\frac{\operatorname{Emax}^{m}}{P^{m}}\right) .
$$

Similar to recharge based solutions, in these cases we propose to iteratively remove from $\mathcal{V}^{*}$ one of the sensors to be replaced until the feasibility conditions are met. In particular, the node with the highest estimated lifetime $L_{i}(t)$ is removed from the set of nodes to recharge $\mathcal{V}^{*}$ (see lines 19-20 in Algorithm 3). Obviously, this is an emergency situation where the proposed solution is implemented in the hope that the removed sensors will somehow survive (e.g. the lifetime value was a pessimistic estimation of the real value).

- Case 2: the robot has not sufficient nodes to replace all endangered senors:

$$
\operatorname{Nmax}{ }^{r}<\left|\mathcal{V}^{*}\right|
$$

Also in this case we propose to iteratively remove one of the sensors to be replaced until the feasibility conditions are met (we fall again in the condition stated in lines 1920 in Algorithm 3).

- Case 3: the time available to replace the sensors is not enough to replace them all:

$$
\left(T-T^{\text {travel }}\left(\mathcal{V}^{*}\right)\right)<\left(\left|\mathcal{V}^{*}\right| \cdot T^{r}\right) .
$$

As for the other problematic cases one or more nodes will be removed from $\mathcal{V}^{*}$ until the feasibility conditions are met (same condition stated in lines 19-20 in Algorithm 3).

- Case 4: the time available to replace the sensors is enough to replace them all and the number of available replacement sensors is sufficient:

$$
\left(\left(T-T^{\text {travel }}\left(\mathcal{V}^{*}\right)\right) \geq\left(\left|\mathcal{V}^{*}\right| \cdot T^{r}\right)\right) \wedge\left(\operatorname{Nmax}^{r} \geq\left|\mathcal{V}^{*}\right|\right) .
$$

In this case, all nodes in $\mathcal{V}^{*}$ are replaced and the mobile robot moves back to the base station for the next maintenance cycle (see lines 21-23 in Algorithm 3).

\subsection{Distributed Approach for Node Replacement}

\subsubsection{Nodes visited for maintenance}

A mobile robot makes stepwise decisions based on the information gathered from the nodes in its sensing range. A difference from the recharge based approach is that now a threshold value is set on the residual energy to consider a node "in need of replacement". This avoids the inefficient behavior of a robot replacing sensors with almost full battery. The threshold value we consider in this analysis is two times the minimum energy for sensing $2 \cdot \operatorname{Emin}_{i}$ (see Algorithm (4)). However, this value could be tuned according to the operational scenario, to improve the efficiency of the replacement algorithm. 


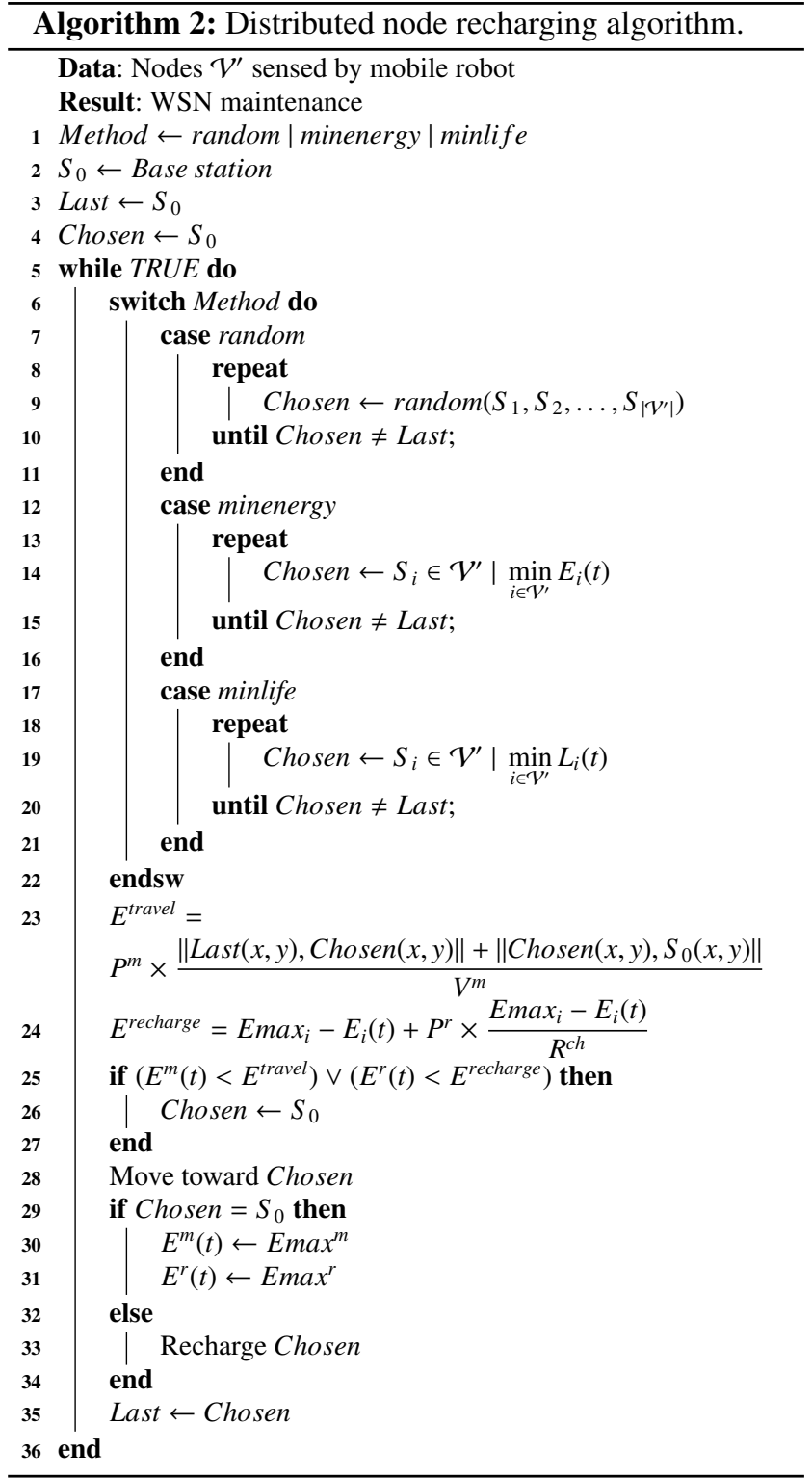

\subsubsection{Node visiting order}

Also in this case, three algorithms, which drive the decisions taken by the mobile robot, are proposed: the so-called random, minenergy, and minlife approaches already presented in section 5.2 (see lines 1-22 in Algorithm 4). The remainder of the algorithm is the same as the one in the recharging case, but for the condition set on the number of replacing sensors available at the mobile robot (see line 24 in Algorithm 4.

\section{Performance Evaluation}

In this section we present simulation results relevant to the proposed node recharging and replacement policies for WSN

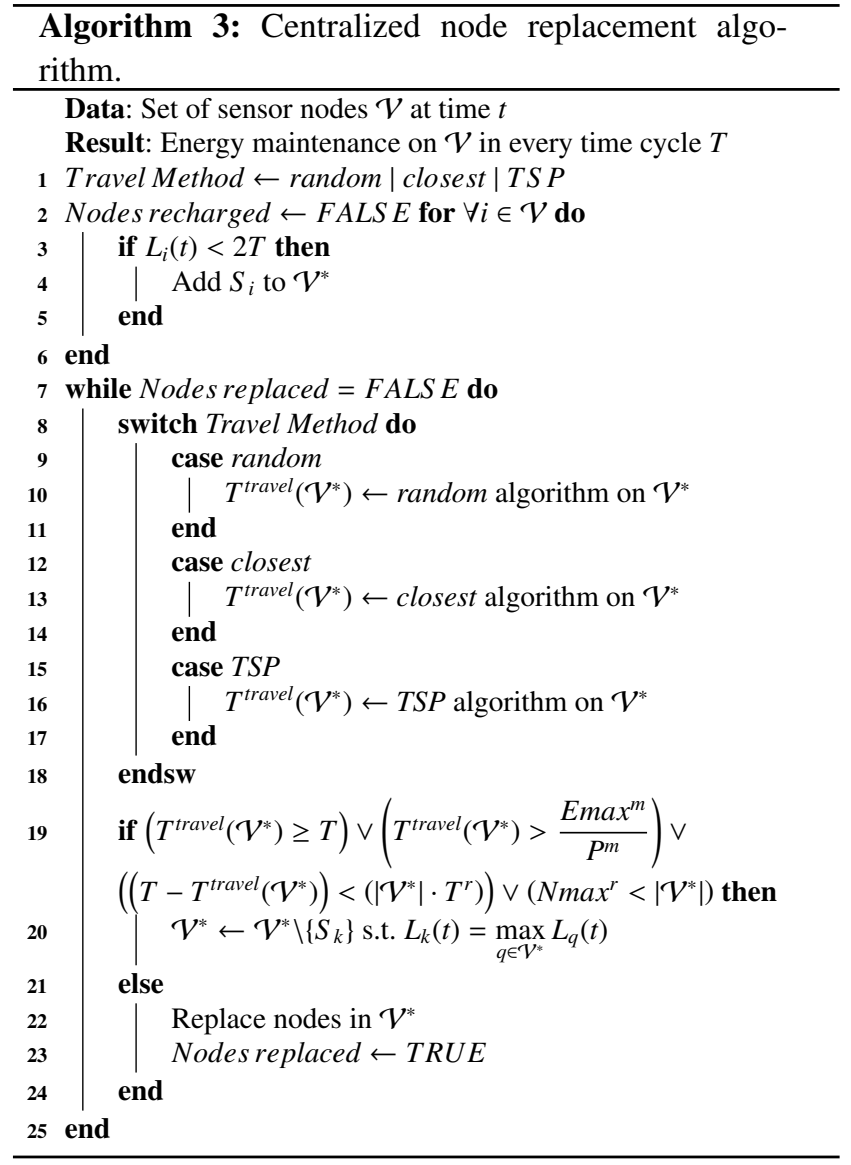

maintenance. The key performance parameters in our analysis are:

- Sustainability ratio: given a number of simulation runs, this parameter shows the number of times the objective of maintaining all sensors alive is reached;

- Network lifetime: that is the average value expressed in hours, between the network start-up and the first death of a sensor in the network;

- Percentage of dead nodes: this gives a quantitative value of the number of dead nodes, in those case where the network maintenance algorithm did not succeed to keep the network alive;

- Movement energy: expressed in kilojoule (kJ), it captures the amount of energy spent by the mobile robot in moving around the network. This parameter will highlight how efficient, in terms of energy consumption, the adopted movement path selection is for the mobile robot.

All scenarios are tested under realistic WSN implementations modeled with the WSNet simulator [21]. A variable number of sensors in the range $20-80$ is considered, randomly deployed within a simulated area of $100 m \times 100 m$. Each simulation run covers 24 hours and the average results for each of the proposed solutions are reported. Further pa- 


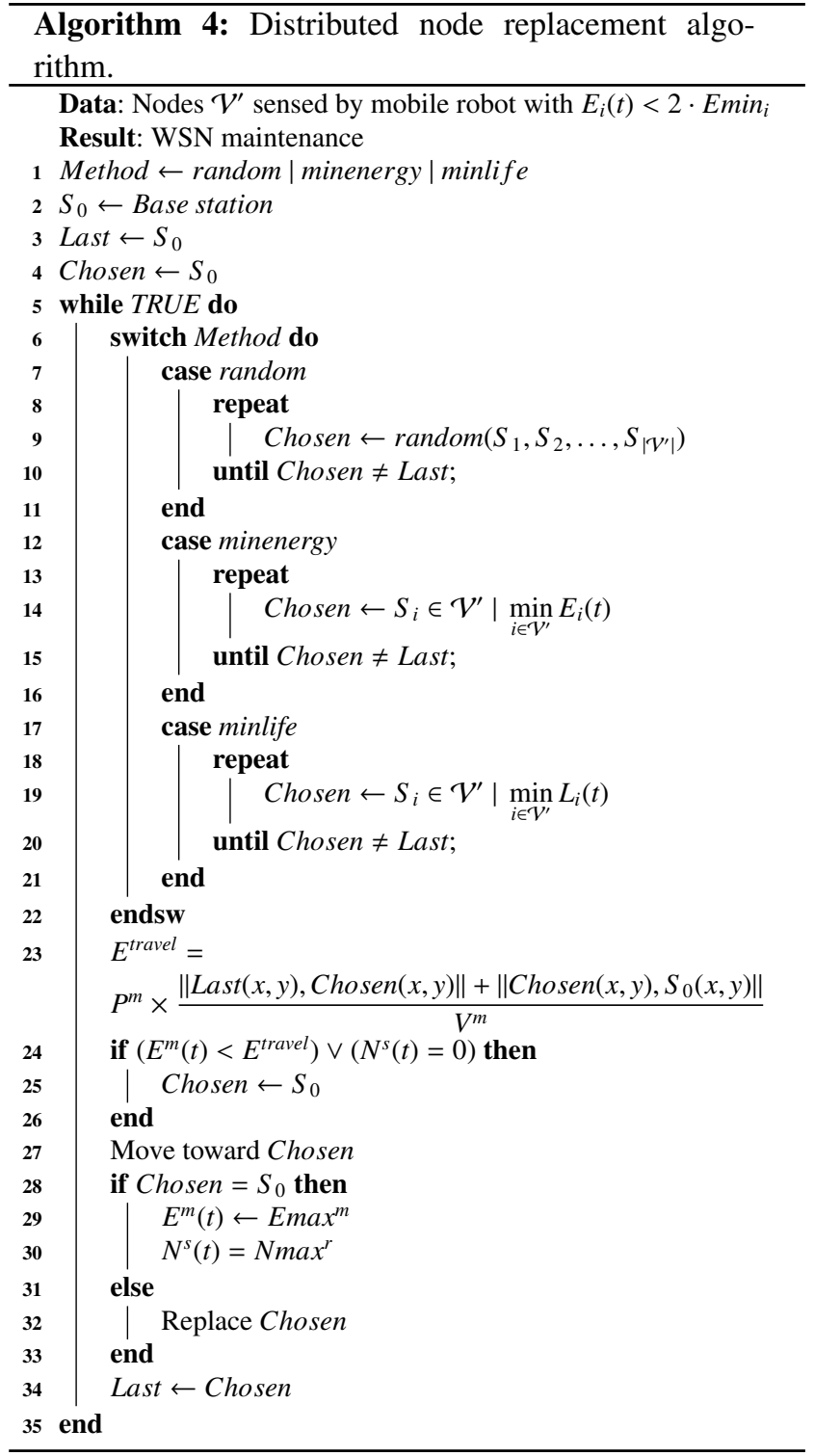

rameter settings for the sensor nodes and the mobile robot are reported in Table 4.

Before showing the behavior of the performance figures of interest, a preliminary analysis is first presented to determine the best time cycle $T$ duration to be adopted for the centralized approaches.

\subsection{Definition of time cycle in centralized approaches}

A set of simulations has been run to experimentally find the optimal value of time period $T$ for the node recharging solution described in section 5 . The analysis has been performed for values of $T \in\{0.5,1,1.5,2,2.5,3\} \mathrm{h}$ in a sample scenario with a random movement technique assumed for the mobile robot and 80 nodes (similar results are obtained for other tested solutions). Fig. 3 shows the results in terms of
Table 4 Simulation parameters.

\begin{tabular}{|c|l|c|}
\hline & Parameter & Value \\
\hline \multirow{5}{*}{ Robot } & Movement energy Emax ${ }^{m}$ & $5000 \mathrm{~kJ}$ \\
& Recharging energy Emax & $2000 \mathrm{~kJ}$ \\
& Movement power consumption $P^{m}$ & $50 \mathrm{~W}$ \\
& Recharging power consumption $P^{r}$ & $3 \mathrm{~W}$ \\
& Movement velocity $V^{m}$ & $1 \mathrm{~m} / \mathrm{s}$ \\
& Energy sources refill $T^{\text {replace }}$ & $0 \mathrm{~s}$ \\
& Sensing range $r^{s}$ & $30 \mathrm{~m}$ \\
& Time needed to replace & $20 \mathrm{~s}$ \\
& a sensor node $T^{r}$ & 50 \\
& Number of sensor & \\
& replacements $N$ max & \\
\hline \multirow{5}{*}{ Sensor $i$} & Information sampling rate $F_{i}$ & $1 \mathrm{~Hz}$ \\
& Sensing power consumption $P_{i}^{s}$ & $0.1 \mathrm{~J} / \mathrm{packet}$ \\
& Transmission power & $0.1 \mathrm{~J} / \mathrm{packet}$ \\
& consumption $P_{i}^{t}$ & \\
& Maximal energy Emax & $9 \mathrm{~kJ}$ \\
& Minimal energy for sensing $\operatorname{Emin}_{i}$ & $0.05 E m a x_{i}[22]$ \\
& Battery recharging rate $R^{c^{c h}}$ & $25 \mathrm{~J} / \mathrm{min}$ \\
& Communication range $r^{c}$ & $30 \mathrm{~m}$ \\
\hline \multirow{5}{*}{ Simulation } & Simulation duration & $24 \mathrm{~h}$ \\
& Simulated area & $100 \mathrm{~m} \times 100 \mathrm{~m}$ \\
& Number of sensors & $20 \ldots 80$ \\
\hline
\end{tabular}

the four main performance figures, namely the percentage of sustainability ratio, dead nodes percentage, network lifetime, and average energy consumed by the mobile robot for movement. Observing the plotted results, we can conclude that a good value to be adopted is $T=1.5 \mathrm{~h}$. This value will be used for the remaining simulations for the assessment of the centralized approaches performance.

\subsection{WSN sustainability ratio analysis}

In this analysis all the proposed solutions are compared in terms of WSN sustainability. The obtained results are plotted in Fig. 4. What emerges is that, for the adopted simulations settings, the replacement techniques perform better than the recharging ones. The motivation for this is that the time needed for node replacement is much lower than the time needed to recharge the sensor nodes, which is a reasonable assumption. In particular, from subplot (b) we observe that in a centralized approach the replacement techniques always succeed to maintain the network alive. In particular, this is true independently from the path selection algorithm for the mobile robot. When looking at subplot (d) instead, we observe that the distributed approach for node replacement is adversely affected by a random choice of the next node to replace. In fact, the percentage of sustainability dramatically decreases with the number of nodes in the network.

When focusing on the recharging techniques in subplots (a) and (c), we notice that the network sustainability is always guaranteed with small networks of 20 sensors. When increasing the number of nodes this value decreases. The 


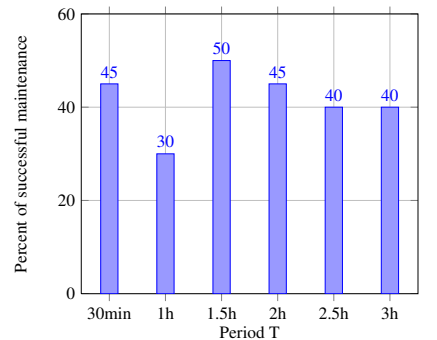

(a) WSN sustainability ratio.

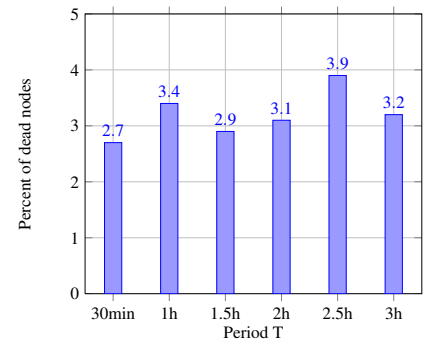

(b) Percent of dead nodes.

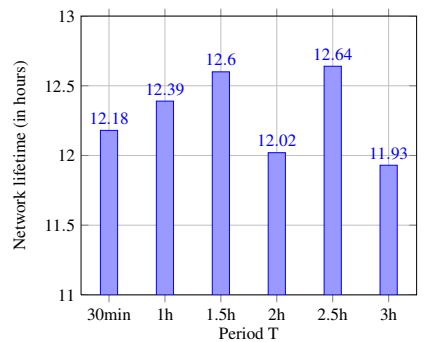

(c) Network lifetime.

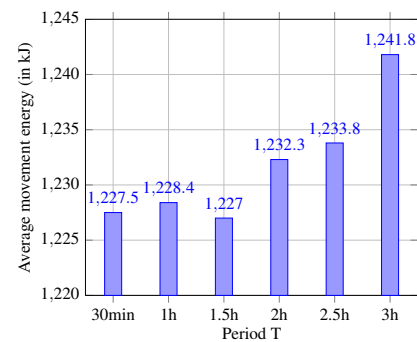

(d) Average movement energy.

Fig. 3 Evaluation metrics for centralized approach to node recharging with random technique, 80 nodes and period $T \in$ $\{30 \min , 1 h, 1.5 h, 2 h, 2.5 h, 3 h\}$.

distributed approach, for any movement solution of the mobile robot, with 40 nodes and above never succeeds in keeping the network alive, see subplot (c). For the centralized approach reported in subplot (a), we observe that the movement of the mobile robot has some influence with 40 nodes. In particular, the path selection based on the TS P solution performs the best with about $45 \%$ of network sustainability while the so-called closest algorithm guarantees about $20 \%$ of network sustainability.

Noteworthy, for node replacement techniques, increasing the number of sensors will at some point provoke the non-sustainability of the network.

\subsection{Network lifetime analysis}

Let us now further investigate on the average lifetime of the WSN in the simulated scenarios. For those cases where the network sustainability is always guaranteed the network lifetime is obviously equal to the whole simulation time. This happens: for all centralized replacement techniques in subplot (b) in Fig. 5, for the distributed replacement techniques in subplot (d) with minenergy and minlife robot movement solutions, and for all the recharging solutions with 20 nodes. For the random robot movement solution instead, the network lifetime is reduced to about 4,5 hours already for 40 nodes.

Besides, for the centralized recharging approach with 40 nodes, in subplot (a) we can observe that the TS $P$ guarantees longer network lifetime on average, followed by the closest and the random solutions. A clearer distinction among the movement solutions for the mobile robot, can be observed in subplot (c) for the distributed recharging solution. In fact, for all values of the number of nodes in the network larger than 20, the minenergy solution guarantees longer network lifetime than the minlife and the random solutions. The observed differences are related to how efficient the robot movement selection is for the considered solutions. The more the robot movement is efficient, the longer the network can be kept alive.

\subsection{Percentage of dead nodes analysis}

As plotted in Fig. 6, in those cases where the network sustainability is always guaranteed, the percentage of dead nodes is obviously equal to zero (refer to replacement techniques in subplots (b) and (d)). For the replacement technique, only a distributed approach with random movement of the mobile robot makes the percentage of dead nodes increasing with the total number of nodes in the WSN. This happens because the choices of the mobile robot become less effective as the network size increases. More interesting is the analysis of the recharging approaches in subplot (a) and (c). For the centralized approach in subplot (a), we observe that, as expected, the TSP is performing the best, followed by the closest solution based on local distances between nodes and the random solutions. For the distributed approach instead (subplot (c)) we observe that starting from 40 nodes the minenergy and minlife algorithms seem to introduce more dead nodes than the random solution. In particular, the plots show that a local distributed decision based on local information on the energy-related constraints of single nodes does not actually improve the global performance and the network is not kept alive (please refer to the plots in Fig. 4).

\subsection{Movement energy for the mobile robot analysis}

In this last analysis, we investigate on how the proposed movement path selection algorithms influence the energy consumption for the mobile robot. Focusing the attention on the centralized approaches, subplots (a) and (b) in Fig. 7 , the average movement energy for the mobile robot is always the highest for the random algorithm. It has the lowest 


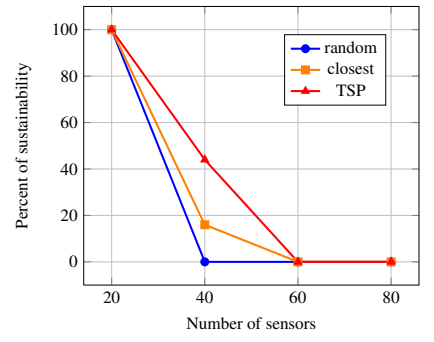

(a) Centralized recharging

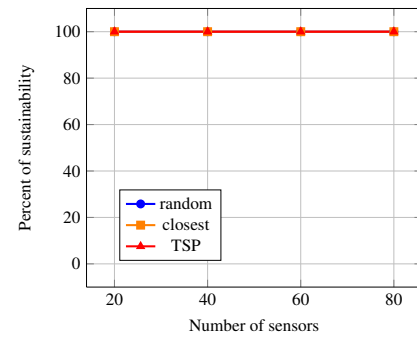

(b) Centralized replacement

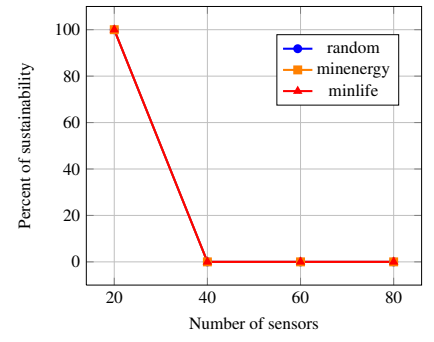

(c) Distributed recharging

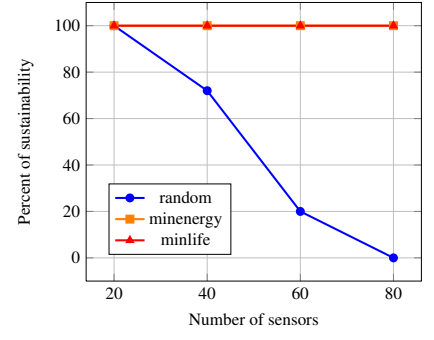

(d) Distributed replacement

Fig. 4 Percent of successful maintenance.

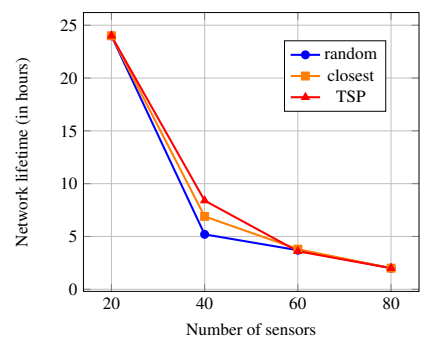

(a) Centralized recharging

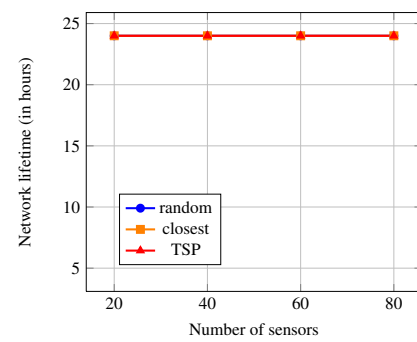

(b) Centralized replacement

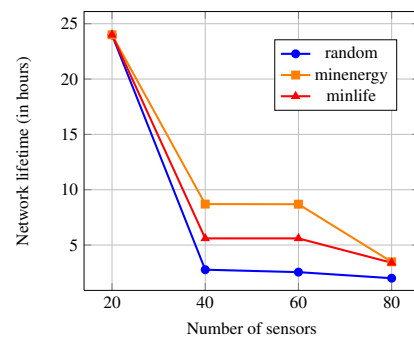

(c) Distributed recharging

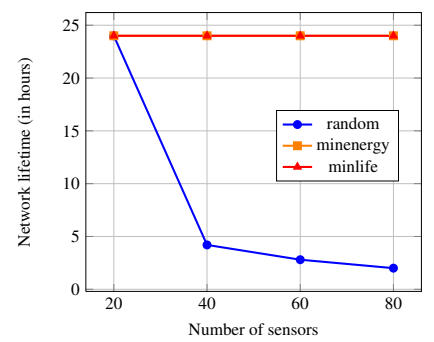

(d) Distributed replacement

Fig. 5 Network lifetime achieved during the maintenance.

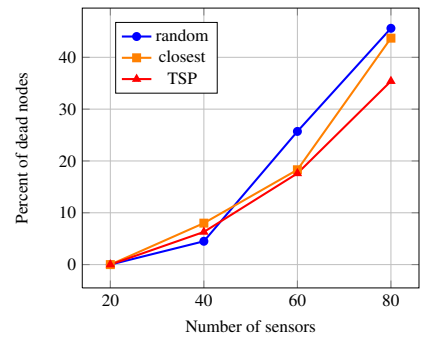

(a) Centralized recharging

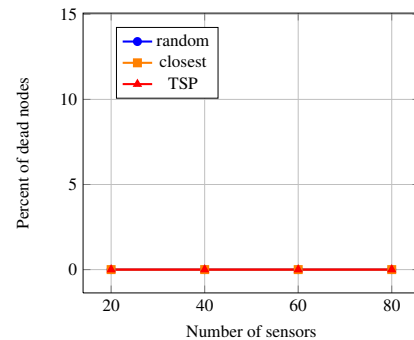

(b) Centralized replacement

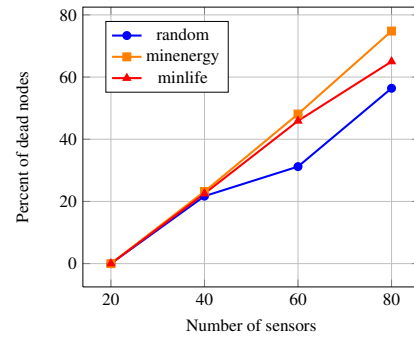

(c) Distributed recharging

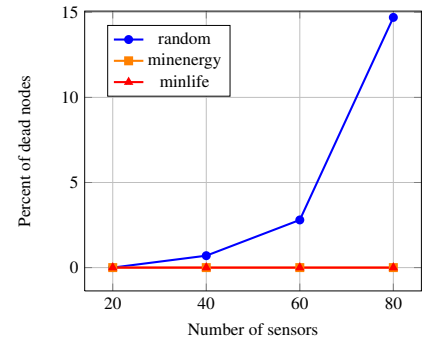

(d) Distributed replacement

Fig. 6 Percent of dead nodes during the maintenance.

value for the TSP, while the closest algorithm shows values in between the other two solutions. For the distributed approaches, see subplots (c) and (d), again the random algorithm is the worst performing solution. Concerning the other two solutions, the differences between minenergy and the minlife solutions are more evident with node replacement, with the minenergy solution showing higher movement energy consumption for the mobile robot. Finally, looking at the recharging based solution, subplots (a) and (c) in Fig. 7 , we underline that for more than 40 nodes the energy for movement is much lower because we do not succeed in keeping the network alive (see analysis in Fig. 4).
From a comparative analysis of the performance figures reported in this section, we can summarize the most important findings in Table 5.

\section{Conclusions}

In this paper we proposed solutions for wireless sensor networks maintenance based on the use of mobile robots visiting the nodes requiring assistance. A careful analysis on the constraints set by the network has been performed to classify the possible solutions based on recharging and re- 


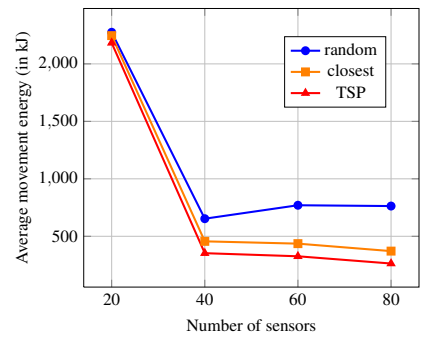

(a) Centralized recharging

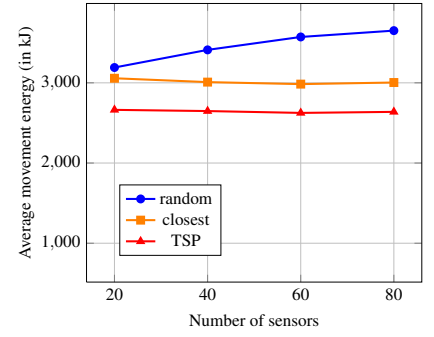

(b) Centralized replacement

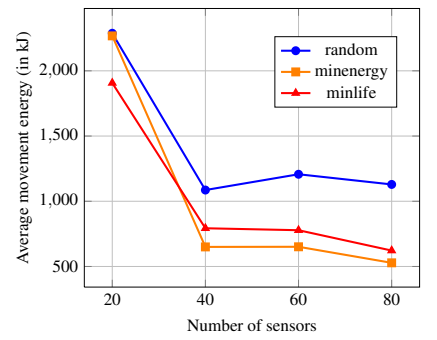

(c) Distributed recharging

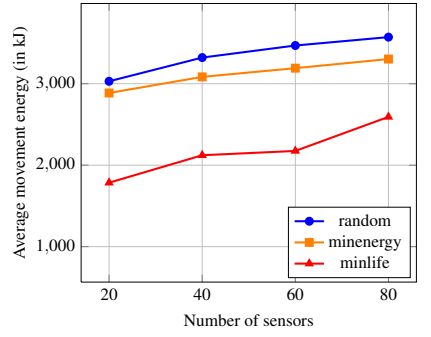

(d) Distributed replacement

Fig. 7 Average movement energy during the maintenance.

Table 5 Summary of main findings.

\begin{tabular}{|l|l|l|l|l|}
\hline & \multicolumn{2}{|c|}{ Centralized } & \multicolumn{2}{c|}{ Distributed } \\
\hline & \multicolumn{1}{|c|}{ Recharging } & Replacing & Recharging & \multicolumn{1}{c|}{ Replacing } \\
\hline \hline $\begin{array}{l}\text { Successful main- } \\
\text { tenance }\end{array}$ & $20-40$ nodes & $20-80$ nodes & 20 nodes & $20-80$ nodes \\
\hline $\begin{array}{l}\text { Most energy effi- } \\
\text { cient path }\end{array}$ & TSP & TSP & minenergy & minlife \\
\hline
\end{tabular}

placement techniques. Both centralized and distributed approaches have been presented, with a cautious view on the possible movement pattern selection for the mobile robot. Further issues addressed in the paper are (i) the definition of parameters to be considered for successful network maintenance, (ii) the identification of possible cases for each solutions as determined by the energy constraints for the nodes and the mobile robot, and (iii) a comparative analysis of movement patterns and maintenance solutions implemented by the mobile robot. The presented performance evaluation gives a preliminary understanding on how and to what extent the proposed solutions succeed in guaranteeing the selfsustainability of a WSN. We believe that the proposed comparative analysis will be very helpful for future research activities in this field.

\section{References}

1. Akyildiz, I., Melodia, T., Chowdhury, K.: A survey on wireless multimedia sensor networks. Computer Networks 51(4), 921-960 (2007)

2. Akyildiz, I., Su, W., Sankarasubramaniam, Y., Cayirci, E.: A survey on sensor networks. Computer Networks 38(4), 393422 (2002)

3. Anastasi, G., Conti, M., Francesco, M.D., Passarella, A.: Energy conservation in wireless sensor networks: a survey. Ad Hoc Networks 7(3) (2009)

4. Chang, J., Tassiulas, L.: Maximum lifetime routing in wireless sensor networks. IEEE/ACM Transactions on Networking 12(4), 609-619 (2004)

5. Kurs, A., Karalis, A., Moffatt, R., Joannopoulos, J., Fisher, P., Soljacic, M.: Wireless power transfer via strongly coupled magnetic resonances. In: Science, vol. 317, pp. 83-86 (2007)
6. Kurs, A., Moffatt, R., Soljacic, M.: Simultaneous mid-range power transfer to multiple devices. In: Appl. Phys. Lett., vol. 96 (2010)

7. Liu, L., Hu, B., Li, L.: Energy conservation algorithms for maintaining coverage and connectivity in wireless sensor networks. IET communications 4(7), 786-800 (2010)

8. Magklara, K., Zorbas, D., Razafindralambo, T.: Node Discovery and Replacement Using Mobile Robot. In: 4th International Conference on Ad Hoc Networks. Paris, France (2012). URL http://hal.inria.fr/hal-00740172

9. Mei, Y., Xian, C., Das, S., Hu, Y.C., Lu, Y.H.: Sensor replacement using mobile robots. Computer Communications 30(13), 2615-2626 (2007). DOI 10.1016/j.comcom.2007.05.047. URL http://dx.doi.org/10.1016/j.comcom.2007.05.047

10. Padberg, M., Rinaldi, G.: A branch-and-cut algorithm for the resolution of large-scale symmetric traveling salesman problems. SIAM Review 14(1), 60-100 (1991)

11. Pantazis, N.A., Vergados, D.D.: A Survey on Power Control Issues in Wireless Sensor Networks. IEEE Communications Surveys and Tutorials 9(4), 86-107 (2007)

12. Peng, Y., Li, Z., Zhang, W., Qiao, D.: Prolonging sensor network lifetime through wireless charging. In: Real-Time Systems Symposium (RTSS), 2010 IEEE 31st. Iowa State Univ., Ames, IA, USA (2010)

13. Sheu, J.P., Hsieh, K.Y., Cheng, P.W.: Design and implementation of mobile robot for nodes replacement in wireless sensor networks. Journal of Information Science and Engineering (2008)

14. Shi, J., Xie, L., Hou, Y.T., Sherali, H.D.: On renewable sensor networks with wireless energy transfer. In: INFOCOM, 2011 Proceedings IEEE. Virginia Polytech. Inst. and State Univ., Blacksburg, VA, USA (2011)

15. Sudevalayam, S., Kulkarni, P.: Energy harvesting sensor nodes: Survey and implications. In: Communications Surveys and Tutorials, vol. 13, pp. 1-19 (2011)

16. Tong, B., Li, Z., Wang, G., Zhang, W.: On-demand node reclamation and replacement for guaranteed area coverage in long-lived sensor networks. QShine '09 (2009)

17. Tong, B., Wang, G., Zhang, W., Wang, C.: Node reclamation and replacement for long-lived sensor networks. SECON (2009) 
18. Wang, G., Cao, G., Porta, T., Zhang, W.: Sensor relation in mobile sensor networks. INFOCOM 4, 2302-2312 (2005)

19. Wang, G., Srinivasan, V., Chua, K.: Extending the lifetime of wireless sensor networks through mobile relays. IEEE/ACM Transactions on Networking 16(5), 1108-1120 (2008)

20. Watteyne, T., Molinaro, A., Richichi, M., Dohler, M.: From MANET To IETF ROLL Standardization: A Paradigm Shift in WSN Routing Protocols. IEEE Communications Surveys and Tutorials 13(4), 688-707 (2011)

21. WSNet: An event-driven simulator for large scale wireless sensor networks, wsnet.gforge.inria.fr. URL wsnet.gforge.inria.fr

22. Xie, L., Shi, J., Hou, Y.T., Sherali, H.D.: Making sensor networks immortal: An energy-renewal approach with wireless power transfer. IEEE/ACM Transactions on Networking (2012)

23. Xie, L., Shi, Y., Hou, Y., Lou, W., Sherali, H., Midkiff, S.: On renewable sensor networks with wireless energy transfer: The multinode case. Sensor, Mesh and Ad Hoc Communications and Networks (SECON) (2012)

24. Yeh, L.W., Wang, Y.C., Tseng, Y.C.: ipower: an energy conservation system for intelligent buildings by wireless sensor networks. International Journal of Sensor Networks 5(1), 1-10 (2009)

25. Yick, J., Mukherjee, B., Ghosal, D.: Wireless sensor network survey. Computer Networks 52, 2292-2330 (2008) 